\title{
Linear maps preserving quasi-commutativity
}

\author{
by \\ Heydar Radjavi (Waterloo, ON) and Peter Šemrl (Ljubljana)
}

\begin{abstract}
Let $X$ and $Y$ be Banach spaces and $\mathcal{B}(X)$ and $\mathcal{B}(Y)$ the algebras of all bounded linear operators on $X$ and $Y$, respectively. We say that $A, B \in \mathcal{B}(X)$ quasicommute if there exists a nonzero scalar $\omega$ such that $A B=\omega B A$. We characterize bijective linear maps $\phi: \mathcal{B}(X) \rightarrow \mathcal{B}(Y)$ preserving quasi-commutativity. In fact, such a characterization can be proved for much more general algebras. In the finite-dimensional case the same result can be obtained without the bijectivity assumption.
\end{abstract}

1. Introduction and statement of the main results. Let $\mathcal{A}$ be an algebra over a field $\mathbb{F}$. We say that two elements $a, b \in \mathcal{A}$ quasi-commute if there exists a nonzero $\omega \in \mathbb{F}$ such that $a b=\omega b a$. For results on quasicommutative matrices we refer to [6], where the Potter-Schützenberger formula for such matrices is derived. This formula is of considerable interest in the study of quantum groups. In [3] and [11] certain sufficient conditions for quasi-commutativity of bounded operators were established and in the first of these two papers applications to quantum mechanics were given.

In this paper we will improve Molnár's characterization of bijective linear maps on matrices preserving quasi-commutativity in both directions [7]. Let $M_{n}$ denote the algebra of all $n \times n$ complex matrices. A linear map $\phi: M_{n} \rightarrow M_{n}$ preserves quasi-commutativity in both directions provided that for every pair $A, B \in M_{n}$ the matrices $A$ and $B$ quasi-commute if and only if $\phi(A)$ and $\phi(B)$ quasi-commute. We say that $\phi$ preserves quasicommutativity if $\phi(A)$ and $\phi(B)$ quasi-commute whenever $A$ and $B$ quasicommute. Molnár proved that every bijective linear map $\phi: M_{n} \rightarrow M_{n}$, $n \geq 2$, which preserves quasi-commutativity in both directions is either an inner automorphism multiplied by a nonzero constant, or an inner antiautomorphism multiplied by a nonzero constant.

2000 Mathematics Subject Classification: 15A04, 15A27, 47B49.

Key words and phrases: linear preserver, quasi-commutativity, commutant, quasicommutant.

The first author was supported by the NSERC of Canada. The second author was supported by a grant from the Ministry of Science of Slovenia. 
We propose to consider the following questions here. Can we extend this result to the infinite-dimensional case? Can we replace the assumption of preserving quasi-commutativity in both directions by the weaker assumption of preserving quasi-commutativity in one direction only? And if we restrict to the finite-dimensional case, what can be said about quasi-commutativity preserving linear maps in the absence of the bijectivity assumption?

When dealing with the infinite-dimensional case we arrived at a somewhat surprising result. Namely, we discovered that such maps can be characterized on algebras that are much more general than operator algebras. And moreover, the condition of preserving quasi-commutativity in one direction can be replaced by a much weaker assumption that commuting pairs are mapped into quasi-commutative pairs. We have the following result.

TheOREm 1.1. Let $\mathcal{A}$ and $\mathcal{B}$ be centrally closed unital prime algebras over a field $\mathbb{F} \neq \mathrm{GF}(3)$. Suppose that char $\mathcal{B} \neq 2$. Let $\phi: \mathcal{A} \rightarrow \mathcal{B}$ be a bijective linear map having the property that $\phi(a)$ and $\phi(b)$ quasi-commute for every commutative pair $a, b \in \mathcal{A}$. If neither $\mathcal{A}$ nor $\mathcal{B}$ satisfies the standard polynomial identity of degree 4 , then

$$
\phi(a)=c \psi(a)+\mu(a), \quad a \in \mathcal{A},
$$

where $c$ is a nonzero scalar, $\psi$ is an isomorphism or an anti-isomorphism of $\mathcal{A}$ onto $\mathcal{B}$, and $\mu$ is a linear mapping from $\mathcal{A}$ into the center of $\mathcal{B}$. In particular, $\phi$ preserves commutativity in both directions.

In the special case of algebras of bounded operators we get the following almost straightforward consequences.

Corollary 1.2. Let $X$ and $Y$ be Banach spaces, $\operatorname{dim} X \geq 3$, and $\mathcal{B}(X)$ and $\mathcal{B}(Y)$ the algebras of all bounded linear operators on $X$ and $Y$, respectively. Let $\phi: \mathcal{B}(X) \rightarrow \mathcal{B}(Y)$ be a bijective linear map having the property that $\phi(A)$ and $\phi(B)$ quasi-commute for every commutative pair $A, B \in \mathcal{B}(X)$. Then either there exist a bounded linear bijective operator $T: X \rightarrow Y$, a (not necessarily bounded) linear functional $\varphi$ on $\mathcal{B}(X)$ and a nonzero complex number $c$ such that

$$
\phi(A)=c T A T^{-1}+\varphi(A) I, \quad A \in \mathcal{B}(X),
$$

or there exist a bounded linear bijective operator $T: X^{\prime} \rightarrow Y$, a (not necessarily bounded) linear functional $\varphi$ on $\mathcal{B}(X)$ and a nonzero scalar $c$ such that

$$
\phi(A)=c T A^{\prime} T^{-1}+\varphi(A) I, \quad A \in \mathcal{B}(X) .
$$

Corollary 1.3. Let $X$ and $Y$ be Banach spaces, $\operatorname{dim} X \geq 3$, and $\mathcal{B}(X)$ and $\mathcal{B}(Y)$ the algebras of all bounded linear operators on $X$ and $Y$, respectively. Let $\phi: \mathcal{B}(X) \rightarrow \mathcal{B}(Y)$ be a bijective linear map preserving quasicommutativity. Then either there exist a bounded linear bijective operator 
$T: X \rightarrow Y$ and a nonzero complex number $c$ such that

$$
\phi(A)=c T A T^{-1}, \quad A \in \mathcal{B}(X),
$$

or there exist a bounded linear bijective operator $T: X^{\prime} \rightarrow Y$ and a nonzero scalar $c$ such that

$$
\phi(A)=c T A^{\prime} T^{-1}, \quad A \in \mathcal{B}(X) .
$$

The last result can be improved in the finite-dimensional case by removing the bijectivity assumption. However, in this case the map $\phi$ can be degenerate. To explain this notion we need some more definitions. Let $\mathcal{A}$ be an algebra over a field $\mathbb{F}$ and $\mathcal{V} \subset \mathcal{A}$ a linear subspace. We say that $\mathcal{V}$ is commutative if every pair of elements $a, b \in \mathcal{V}$ commute. If for every pair $a, b \in \mathcal{V}$ we can find a nonzero $\omega \in \mathbb{F}$ such that $a b=\omega b a$, then we say that $\mathcal{V}$ is quasi-commutative. Further, $\mathcal{V}$ is said to be square-zero if $a^{2}=0$ for every $a \in \mathcal{V}$. If $\mathcal{V}$ is square-zero, then it is quasi-commutative. Indeed, if $a, b \in \mathcal{V}$, then $0=(a+b)^{2}=a b+b a$. Thus, each pair $a, b \in \mathcal{V}$ anti-commutes, that is, $a b=-b a$. The converse is true if $\operatorname{char} \mathcal{A} \neq 2$. Indeed, let $\mathcal{V} \subset \mathcal{A}$ be an anti-commutative subspace. Then $a^{2}=-a^{2}$ for every $a \in \mathcal{V}$, and thus $a^{2}=0$. Clearly, every linear map $\phi: \mathcal{A} \rightarrow \mathcal{A}$ whose image is either commutative or square-zero preserves quasi-commutativity. We will show that in the matrix case every linear map preserving quasi-commutativity is either bijective, or its image is commutative or square-zero.

Theorem 1.4. Let $\phi: M_{n} \rightarrow M_{n}, n \geq 3$, be a linear map. Assume that $\phi(A)$ and $\phi(B)$ quasi-commute for every pair of quasi-commuting matrices $A$ and $B$. Then we have one of the following three possibilities:

(1) The range of $\phi$ is commutative.

(2) The range of $\phi$ is anti-commutative.

(3) There exist an invertible $T \in M_{n}$ and a nonzero complex number $c$ such that either

$$
\phi(A)=c T A T^{-1}, \quad A \in M_{n}
$$

or

$$
\phi(A)=c T A^{t} T^{-1}, \quad A \in M_{n} .
$$

Here, $A^{t}$ denotes the transpose of $A$.

TheOrem 1.5. Let $\phi: M_{2} \rightarrow M_{2}$ be a linear map. Then $\phi$ maps the set of quasi-commuting pairs into itself if and only if one of the following four possibilities occurs:

(1) The range of $\phi$ is commutative.

(2) There exist an invertible $T \in M_{2}$ and a nonzero scalar $c$ such that either

$$
\phi(A)=c T A T^{-1}, \quad A \in M_{2},
$$


or

$$
\phi(A)=c T A^{t} T^{-1}, \quad A \in M_{2} .
$$

(3) There exist an invertible $T \in M_{2}$ and a nonzero scalar $c$ such that either

$$
\phi(A)=c\left(T A T^{-1}-(\operatorname{tr} A) I\right), \quad A \in M_{2}
$$

or

$$
\phi(A)=c\left(T A^{t} T^{-1}-(\operatorname{tr} A) I\right), \quad A \in M_{2} .
$$

(4) There exist an invertible $T \in M_{2}$ and a nonzero scalar $c$ such that either

$$
\phi(A)=c\left(T A T^{-1}-\frac{1}{2}(\operatorname{tr} A) I\right), \quad A \in M_{2},
$$

or

$$
\phi(A)=c\left(T A^{t} T^{-1}-\frac{1}{2}(\operatorname{tr} A) I\right), \quad A \in M_{2} .
$$

Here, $\operatorname{tr} A$ denotes the trace of $A$.

We conclude this section by giving some examples of square-zero matrix spaces that are not commutative (such a space is a trivial Jordan algebra and hence simultaneously triangularizable by Jacobson's theorem). Let

$$
N=\left[\begin{array}{ll}
0 & 1 \\
0 & 0
\end{array}\right] \text {. }
$$

Then the set of all $6 \times 6$ matrices of the form

$$
\left[\begin{array}{ccc}
\lambda N & 0 & \mu I \\
0 & \tau N & 0 \\
0 & 0 & -\lambda N
\end{array}\right]
$$

is a 3-dimensional square-zero space which is not commutative. Further, if $\mathcal{V} \subset M_{n}$ and $\mathcal{W} \subset M_{m}$ are non-trivial square-zero subspaces, then the set of all $(2 n+m) \times(2 n+m)$ matrices of the form

$$
\left[\begin{array}{ccc}
A & 0 & \mu I \\
0 & B & 0 \\
0 & 0 & -A
\end{array}\right], \quad A \in \mathcal{V}, B \in \mathcal{W}
$$

is a square-zero space that is not commutative.

An interested reader can find an extensive bibliography in the mainly expository article [10].

\section{Preliminary results}

LEMma 2.1. Let $\mathcal{A}$ be an algebra over an arbitrary field $\mathbb{F}$, char $\mathcal{A} \neq 2$, and $\mathcal{V} \subset \mathcal{A}$ a linear subspace. Assume that $\mathcal{V}$ is quasi-commutative but not commutative. Then $\mathcal{V}$ is square-zero.

Proof. Assume first that $\mathcal{V}$ is two-dimensional. By our assumptions there exist $x, y \in \mathcal{V}$ and a nonzero $\omega \in \mathbb{F}, \omega \neq 1$, such that $x y=\omega y x \neq 0$. Clearly, 
$\mathcal{V}$ is the linear span of $x$ and $y$, and therefore, we have to show that $a^{2}=0$ for every $a=\lambda x+\mu y, \lambda, \mu \in \mathbb{F}$. We may assume that at least one of the scalars $\lambda, \mu$, say $\lambda$, is nonzero. Then there is no loss of generality in assuming that $\lambda=1$.

Thus, $a=x+\mu y$. It is trivial to see that $a$ and $y$ do not commute. Indeed, if $x+\mu y$ and $y$ commuted, then $x$ and $y$ would commute, a contradiction. It follows that $y a=\eta a y \neq 0$ for some $\eta \neq 1$.

We further know that

$$
(a+y) a=\xi a(a+y)
$$

for some nonzero $\xi$. Again, $\xi \neq 1$, since otherwise $a$ and $y$ would commute. Hence,

$$
(1-\xi) a^{2}=(\xi-\eta) a y .
$$

We must show that the assumption $a^{2} \neq 0$ leads to a contradiction. Indeed, assume that $a$ is not square-zero. Then

$$
a^{2}=\tau a y
$$

with $\tau=(1-\xi)^{-1}(\xi-\eta) \neq 0$. Since $a-\tau y$ and $a$ quasi-commute we can find a nonzero $\delta$ such that

$$
a(a-\tau y)=\delta(a-\tau y) a .
$$

But the left-hand side of this equality is equal to 0 , and consequently, $a^{2}=\tau y a$. This together with $a^{2}=\tau a y$ implies that $a$ and $y$ commute, a contradiction.

Let now $\mathcal{V}$ be of any dimension. As before, let $x, y \in \mathcal{V}$ and $\omega \neq 0,1$ satisfy $x y=\omega y x \neq 0$. Assume further that there exists $a \in \mathcal{V}$ such that $a^{2} \neq 0$. Then $a$ commutes with every $b \in \mathcal{V}$, since otherwise the linear span of $a$ and $b$ would be a two-dimensional quasi-commutative subspace that is not commutative, which would imply by the previous step that $a^{2}=0$, a contradiction.

Take any $c \in \mathcal{V}$. If $d \in \mathcal{V}$ is any element that does not commute with $c$, then by the previous step applied to the linear span of $c$ and $d$, we get $c^{2}=d^{2}=(c+d)^{2}=0$, which further implies that $c$ and $d$ anti-commute. It follows that $\mathcal{V}$ is the union of the subset of all elements of $\mathcal{V}$ that commute with $c$ and the subset of all elements of $\mathcal{V}$ that anti-commute with $c$. Both these subsets are actually subspaces. A linear space cannot be a union of two proper subspaces. So, we have proved that for every $c \in \mathcal{V}$, either $c$ commutes with every element of $\mathcal{V}$, or $c$ anti-commutes with every element of $\mathcal{V}$. In particular, since $x$ and $y$ do not commute, we have $x a=-a x$. On the other hand, we already know that a commutes with every element of $\mathcal{V}$, and thus,

$$
a x=x a=0 .
$$


We will now show that $a+x$ commutes with every element of $\mathcal{V}$. Indeed, the other possibility would be that $a+x$ anti-commutes with every element of $\mathcal{V}$, which would imply that $(a+x) a=-a(a+x)$. It would follow that $a^{2}=0$, a contradiction. In a similar way we show that $a+y$ commutes with every element of $\mathcal{V}$ and $a y=y a=0$. Hence,

$$
a^{2}+x y=(a+x)(a+y)=(a+y)(a+x)=a^{2}+y x,
$$

and therefore, $x y=y x$. This contradiction completes the proof.

From this result we can get almost directly the following property of linear maps sending commuting pairs into quasi-commuting pairs. To formulate this corollary we need two more definitions. Let $\mathcal{A}$ be an algebra and $a \in \mathcal{A}$. Then we will denote by $a^{\prime}$ and $a^{-}$the commutant of $a$ and the anti-commutant of $a$, respectively,

$$
a^{\prime}=\{b \in \mathcal{A}: a b=b a\}, \quad a^{-}=\{b \in \mathcal{A}: a b=-b a\} .
$$

Corollary 2.2. Let $\mathcal{A}$ and $\mathcal{B}$ be algebras over an arbitrary field $\mathbb{F}$, $\operatorname{char} \mathcal{B} \neq 2$, and let $\phi: \mathcal{A} \rightarrow \mathcal{B}$ be a linear map. Assume that $\phi(a)$ and $\phi(b)$ quasi-commute for every pair of elements $a, b \in \mathcal{A}$ satisfying $a b=b a$. Then for every $a \in \mathcal{A}$ we have

$$
\phi\left(a^{\prime}\right) \subset(\phi(a))^{\prime} \quad \text { or } \quad \phi\left(a^{\prime}\right) \subset(\phi(a))^{-} .
$$

Proof. Choose any $b \in \mathcal{A}$ that commutes with $a$. Then, clearly, any two linear combinations of $a$ and $b$ commute. If $\phi(a)$ and $\phi(b)$ are linearly dependent, then they commute. Otherwise, the linear span of these two vectors is a two-dimensional quasi-commutative subspace of $\mathcal{B}$. By the previous lemma we have either $\phi(a) \phi(b)=\phi(b) \phi(a)$ or $\phi(a) \phi(b)=-\phi(b) \phi(a)$. It follows that

$$
\phi\left(a^{\prime}\right) \subset(\phi(a))^{\prime} \cup(\phi(a))^{-} .
$$

Clearly, if a linear subspace is contained in the union of two linear subspaces, then it is already contained in one of these two subspaces. This completes the proof.

\section{Proofs of the main results}

Proof of Theorem 1.1. By Corollary 2.2 we have

$$
\phi(\mathcal{A})=\phi\left(1^{\prime}\right) \subset(\phi(1))^{\prime} \quad \text { or } \quad \phi(\mathcal{A}) \subset(\phi(1))^{-} .
$$

Let us first consider the second possibility. By bijectivity we can find $b \in \mathcal{A}$ such that $\phi(b)=1$. Since $\phi(b)$ and $\phi(1)$ anti-commute, we have

$$
0=\phi(1) \phi(b)+\phi(b) \phi(1)=2 \phi(1) .
$$

Thus, $\phi(1)=0$, contradicting the bijectivity of $\phi$.

It follows that we have the first possibility, that is, $\phi(1)$ and $\phi(a)$ commute for every $a \in \mathcal{A}$. In the next step we will show that $\phi$ preserves commu- 
tativity, that is, $\phi(a)$ and $\phi(b)$ commute whenever $a$ and $b$ commute. So, let $a, b \in \mathcal{A}$ be any commutative pair, $a \neq 0$ and $b \neq 0$. Then the linear span of $\phi(a)$ and $\phi(b)$ is a quasi-commutative subspace, and consequently, $\phi(a)$ and $\phi(b)$ commute or anti-commute. We have to show that the first possibility holds. Assume on the contrary that $\phi(a)$ and $\phi(b)$ do not commute.

We further know that $\phi(1+a)$ and $\phi(b)$ commute or anti-commute. In the first case we have

$$
(\phi(1)+\phi(a)) \phi(b)=\phi(b)(\phi(1)+\phi(a)),
$$

which together with the commutativity of the pair $\phi(1), \phi(b)$ implies that $\phi(a)$ and $\phi(b)$ commute, a contradiction. Thus,

$$
(\phi(1)+\phi(a)) \phi(b)=-\phi(b)(\phi(1)+\phi(a)),
$$

which implies that $\phi(1) \phi(b)+\phi(b) \phi(1)=2 \phi(1) \phi(b)=0$. As $\mathcal{B}$ is a unital prime algebra not of characteristic two and since $\phi(1) c \phi(b)=c \phi(1) \phi(b)=0$ for every $c \in \mathcal{B}$, we have $\phi(1)=0$ or $\phi(b)=0$, contradicting the bijectivity of $\phi$.

Hence, $\phi$ preserves commutativity. Our result now follows directly from Brešar's characterization of bijective commutativity preserving maps [1].

Proof of Corollary 1.2. Since $\operatorname{dim} X \geq 3$ and $\phi$ is bijective, we have $\operatorname{dim} Y \geq 3$. Thus, neither of the algebras $\mathcal{B}(X)$ and $\mathcal{B}(Y)$ satisfies the standard polynomial identity of degree 4 . It is easy to see that these two algebras satisfy all other assumptions on $\mathcal{A}$ and $\mathcal{B}$ in Theorem 1.1. Thus, we can apply Theorem 1.1. It is well-known that the center of $\mathcal{B}(Y)$ is the set of all scalar operators and that every isomorphism or anti-isomorphism of $\mathcal{B}(X)$ onto $\mathcal{B}(Y)$ is spatially implemented. This completes the proof.

Proof of Corollary 1.3. We can apply Corollary 1.2. After composing $\phi$ with an appropriate isomorphism or anti-isomorphism of $\mathcal{B}(Y)$ onto $\mathcal{B}(X)$ and multiplying the resulting map by a nonzero constant we may and will assume that $\phi$ maps $\mathcal{B}(X)$ onto itself and

$$
\phi(A)=A+\varphi(A) I, \quad A \in \mathcal{B}(X),
$$

for some linear functional $\varphi$ on $\mathcal{B}(X)$. All we have to do is to show that $\varphi=0$.

We will first show that $\varphi(A)=0$ for every finite rank operator $A \in \mathcal{B}(X)$. Let $B$ be any nilpotent of rank 2 and of nilindex 3 . Then we can write

$$
B=\left[\begin{array}{lll}
0 & 1 & 0 \\
0 & 0 & 1 \\
0 & 0 & 0
\end{array}\right] \oplus 0 .
$$


If we set

$$
A=\left[\begin{array}{lll}
1 & 0 & 0 \\
0 & 2 & 0 \\
0 & 0 & 4
\end{array}\right] \oplus 0
$$

then $A$ and $B$ quasi-commute, and thus $\phi(A)=A+\varphi(A) I$ and $\phi(B)=$ $B+\varphi(B) I$ quasi-commute. Obviously, $A B, A, B, I$ are linearly independent, and one can then easily see that $\phi(A)=A+\varphi(A) I$ and $\phi(B)=B+\varphi(B) I$ quasi-commute only if $\varphi(A)=\varphi(B)=0$. Thus, $\varphi$ is zero on the set of all nilpotents of rank 2 and of nilindex 3 , and by linearity, $\varphi$ must be zero on the set of all nilpotents of rank one. Applying linearity once more we conclude that $\varphi$ is the zero functional on the space of all finite rank trace zero operators. The space of all finite rank operators is a direct sum of the space of all finite rank trace zero operators and a one-dimensional space spanned by $C$, where $C$ is any finite rank operator with nonzero trace. As $\varphi(A)=0$ we conclude that $\varphi(F)=0$ for every finite rank $F \in \mathcal{B}(X)$.

Now let $A \in \mathcal{B}(X)$ be any operator. We decompose $X$ into a direct sum $X=X_{1} \oplus X_{2}$ where $X_{1}$ is 2 -dimensional. With respect to this direct sum decomposition the operator $A$ can be represented as

$$
A=\left[\begin{array}{ll}
A_{1} & A_{2} \\
A_{3} & A_{4}
\end{array}\right]
$$

where $A_{2}: X_{2} \rightarrow X_{1}, A_{3}: X_{1} \rightarrow X_{2}, A_{4}: X_{2} \rightarrow X_{2}$ are bounded linear operators and $A_{1}$ can be represented as a $2 \times 2$ matrix. Define finite rank operators $R, T \in \mathcal{B}(X)$ by

$$
R=\left[\begin{array}{cc}
-A_{1}+R_{1} & -A_{2} \\
-A_{3} & 0
\end{array}\right] \quad \text { and } \quad T=\left[\begin{array}{cc}
T_{1} & 0 \\
0 & 0
\end{array}\right]
$$

where

$$
R_{1}=\left[\begin{array}{cc}
1 & 0 \\
0 & -1
\end{array}\right] \text { and } T_{1}=\left[\begin{array}{cc}
0 & 1 \\
-1 & 0
\end{array}\right]
$$

Then $A+R$ anti-commutes with $T$, and consequently, $A+R+\varphi(A) I$ quasicommutes with $T$. A straightforward computation shows that this is possible only if $\varphi(A)=0$. As $A$ was an arbitrary operator, we have shown that $\varphi=0$.

Proof of Theorem 1.4. We distinguish two cases. First we assume that the set

$$
\left\{A \in M_{n}: \phi(A) \text { commutes with } \phi\left(A^{2}\right)\right\}
$$

is dense in $M_{n}$. Thus, by continuity, $\phi(A)$ commutes with $\phi\left(A^{2}\right)$ for all $A \in M_{n}$. By [2, Lemma 4.3], either the range of $\phi$ is commutative (in which case we are done), or there exist an invertible matrix $T$, a nonzero complex number $c$, and a linear functional $f$ on $M_{n}$ such that either

$$
\phi(A)=c T A T^{-1}+f(A) I, \quad A \in M_{n},
$$


or

$$
\phi(A)=c T A^{\mathrm{t}} T^{-1}+f(A) I, \quad A \in M_{n} .
$$

We must show that $f=0$. We can assume, without loss of generality, that $c=1, T=I$, and

$$
\phi(A)=A+f(A) I
$$

after composing $\phi$ with transposition if necessary.

To show that $f(N)=0$ for an arbitrary nilpotent of rank one, it suffices to verify $f\left(E_{12}\right)=0$. (Here $\left\{E_{i j}\right\}$ denotes the standard basis for $M_{n}$.) Let $A=2 E_{11}+E_{22}$. Then $A E_{12}=2 E_{12} A$, and thus the two matrices $A+f(A) I$ and $E_{12}+f\left(E_{12}\right) I$ quasi-commute:

$$
(A+f(A) I)\left(E_{12}+f\left(E_{12}\right) I\right)=\lambda\left(E_{12}+f\left(E_{12}\right) I\right)(A+f(A) I)
$$

for some $\lambda \neq 0$. Since $I, E_{12}$, and $A$ are linearly independent, it follows that

$$
2+f(A)=\lambda(1+f(A))
$$

and hence $\lambda \neq 1$. But then we also have

$$
f\left(E_{12}\right) A=\lambda f\left(E_{12}\right) A
$$

and thus $f\left(E_{12}\right)=0$.

Since the nilpotents of rank one span the trace-zero matrices, we deduce that $f(X)=\alpha \operatorname{tr} X, X \in M_{n}$, where $\alpha$ is a fixed scalar. Consider a pair of quasi-commuting matrices $A=E_{12}+E_{23}$ and $B=E_{11}-E_{22}+E_{33}$. Now

$$
\phi(A)=A
$$

and

$$
\phi(B)=(\alpha+1) E_{11}+(\alpha-1) E_{22}+(\alpha+1) E_{33}+\alpha\left(I-E_{11}-E_{22}-E_{33}\right)
$$

will quasi-commute only if $\alpha=0$.

To complete the proof, we assume that the set

$$
\left\{A \in M_{n}: \phi(A) \text { commutes with } \phi\left(A^{2}\right)\right\}
$$

is not dense in $M_{n}$. Thus there is a nonempty open set $\mathcal{G}$ such that $\operatorname{span}\{\phi(A)$, $\left.\phi\left(A^{2}\right)\right\}$ is quasi-commutative but not commutative for all $A \in \mathcal{G}$. By Lemma 2.1, $\phi(A)^{2}=0$. Since $\mathcal{G}$ is open, it follows that $\phi(A)^{2}=0$ for all $A \in M_{n}$.

Proof of Theorem 1.5. Once we show that both maps

$$
A \mapsto A-\xi(\operatorname{tr} A) I, \quad \xi \in\{1 / 2,1\},
$$

preserve quasi-commutativity, we can apply the simple fact that the set of quasi-commutativity preserving maps is closed under composition to conclude that any map as in (1), (2), (3), or (4) preserves quasi-commutativity.

Assume that nonzero $A, B \in M_{2}$ quasi-commute. To show that $A-$ $\xi(\operatorname{tr} A) I$ and $B-\xi(\operatorname{tr} B) I$ quasi-commute, we distinguish several cases. We start with the case in which at least one of $A$ and $B$, say $B$, is invertible. 
From $A B=\lambda B A, \lambda \neq 0$, we deduce that $A$ is similar to $\lambda^{n} A$ for all $n$. If $A$ has a nonzero eigenvalue, then $\lambda=1$ or $\lambda=-1$. When $\lambda=1, A$ and $B$ commute, and so do $A-\xi(\operatorname{tr} A) I$ and $B-\xi(\operatorname{tr} B) I$. When $\lambda=-1, A$ is similar to

$$
\left[\begin{array}{cc}
a & 0 \\
0 & -a
\end{array}\right]
$$

with $a \neq 0$. It follows from $A B=-B A$ that the diagonal entries of $B$ are both zero. Thus, $\operatorname{tr} A=\operatorname{tr} B=0$, and we are done. Otherwise $A$ is nilpotent and, without loss of generality, of the form

$$
A=\left[\begin{array}{ll}
0 & 1 \\
0 & 0
\end{array}\right] .
$$

Denote by $A^{\#}$ the quasi-commutant of $A$, that is, $A^{\#}$ is the set of all matrices that quasi-commute with $A$. Then

$$
A^{\#}=\left\{\left[\begin{array}{ll}
x & z \\
0 & y
\end{array}\right]: x y \neq 0 \text { or }|x|+|y|=0\right\} .
$$

For every $B \in A^{\#}$, we clearly have $B-\xi(\operatorname{tr} B) I \in A^{\#}$. Hence $A-\xi(\operatorname{tr} A) I$ and $B-\xi(\operatorname{tr} B) I$ quasi-commute.

We can now assume that $A$ and $B$ are both singular (and nonzero). If they are both nilpotent, then $\operatorname{tr} A=\operatorname{tr} B=0$, and we are done. Otherwise, one of them, say $A$, is a scalar multiple of an idempotent of rank one. Thus, a straightforward calculation shows that $A^{\#}=A^{\prime}$, and we are done again.

We must prove the converse. We first consider the case in which $\phi(I)$ is not a scalar. Then up to similarity, $\phi(I)$ has one of the following forms:

$$
\left[\begin{array}{ll}
a & 1 \\
0 & a
\end{array}\right], \quad\left[\begin{array}{ll}
0 & 1 \\
0 & 0
\end{array}\right], \quad\left[\begin{array}{cc}
b & 0 \\
0 & 0
\end{array}\right], \quad\left[\begin{array}{cc}
c & 0 \\
0 & -c
\end{array}\right], \quad\left[\begin{array}{ll}
d & 0 \\
0 & e
\end{array}\right],
$$

where $a b c d e \neq 0$ and $d \neq \pm e$. Note that $\phi\left(M_{2}\right)$ is contained in $\phi(I)^{\#}$. In the first and third cases $\phi(I)^{\#}=\phi(I)^{\prime}$, which is a commutative subspace, completing the proof for these cases. In the second case we have

$$
\phi\left(M_{2}\right) \subset \phi(I)^{\#}=\left\{\left[\begin{array}{ll}
x & z \\
0 & y
\end{array}\right]: x y \neq 0 \text { or }|x|+|y|=0\right\} .
$$

Since $\phi\left(M_{2}\right)$ is a subspace, there exists a nonzero scalar $k$ such that

$$
\phi\left(M_{2}\right) \subset\left\{\left[\begin{array}{cc}
x & z \\
0 & k x
\end{array}\right]: x, z \in \mathbb{C}\right\} .
$$

If all idempotents are mapped to multiples of $E_{12}$ (i.e., if $x=0$ for all these images), then, since the idempotents span $M_{2}$, the space $\phi\left(M_{2}\right)$ has dimension one and is thus commutative. So we can assume

$$
\phi(E)=\left[\begin{array}{cc}
x_{0} & z_{0} \\
0 & k x_{0}
\end{array}\right], \quad x_{0} \neq 0,
$$


for some idempotent $E$ of rank one. Then

$$
\phi(I-E)=\left[\begin{array}{cc}
-x_{0} & 1-z_{0} \\
0 & -k x_{0}
\end{array}\right] .
$$

Since $\phi(E)$ and $\phi(I-E)$ must necessarily quasi-commute, and since $x_{0} \neq 0$, they actually commute; hence $k=1$, and we are done.

In the last two cases, it is not hard to verify that

$$
\phi\left(M_{2}\right) \subset\left\{\left[\begin{array}{ll}
x & 0 \\
0 & y
\end{array}\right]: x, y \in \mathbb{C}\right\} \cup\left\{\left[\begin{array}{ll}
0 & x \\
y & 0
\end{array}\right]: x, y \in \mathbb{C}\right\} .
$$

Since $\phi\left(M_{2}\right)$ is a subspace contained in the union of the above two subspaces, and since $\phi(I)$ is diagonal, we deduce that $\phi\left(M_{2}\right)$ is contained in the commutative space of diagonal matrices.

We can assume, from now on, that $\phi(I)$ is a scalar matrix.

For our next case, we assume that there is a diagonalizable $A$ whose image is not diagonalizable. By adding an appropriate multiple of $I$ to $A$ if necessary, we can write that, up to similarity,

$$
A=\left[\begin{array}{cc}
\alpha & 0 \\
0 & \beta
\end{array}\right]
$$

with $\alpha \beta \neq 0$. Note that the linear span of $A^{\#}$ is $M_{2}$. By hypothesis, $\phi(A)$ is, again up to similarity,

$$
\left[\begin{array}{ll}
0 & 1 \\
0 & 0
\end{array}\right] \text { or }\left[\begin{array}{ll}
\gamma & 1 \\
0 & \gamma
\end{array}\right]
$$

with $\gamma \neq 0$. In both cases, $\phi(A)^{\#}$ is contained in the space $\mathcal{T}$ of upper triangular matrices. It follows from $\phi\left(A^{\#}\right) \subset \phi(A)^{\#}$ that

$$
\phi\left(M_{2}\right)=\phi\left(\operatorname{span} A^{\#}\right) \subset \operatorname{span} \phi(A)^{\#} \subset \mathcal{T} \text {. }
$$

Our plan now is first to show that if $\phi\left(M_{2}\right)$ is contained in

$$
\left\{\left[\begin{array}{ll}
0 & x \\
0 & y
\end{array}\right]: x, y \in \mathbb{C}\right\} \quad \text { or } \quad\left\{\left[\begin{array}{ll}
y & x \\
0 & 0
\end{array}\right]: x, y \in \mathbb{C}\right\}
$$

then it is actually contained in $\operatorname{span}\left\{E_{12}\right\}$, and secondly, to prove that otherwise there is an invertible diagonalizable $B \in M_{2}$ such that $\phi(B)=a I+b E_{12}$ with $a b \neq 0$. Assuming for a moment that such a $B$ exists, we have

$$
\phi\left(M_{2}\right)=\phi\left(\operatorname{span} B^{\#}\right) \subset \operatorname{span} \phi(B)^{\#}=\phi(B)^{\prime}=\operatorname{span}\left\{I, E_{12}\right\},
$$

and this would complete the proof.

If

then, since

$$
\phi\left(M_{2}\right) \subset\left\{\left[\begin{array}{ll}
0 & x \\
0 & y
\end{array}\right]: x, y \in \mathbb{C}\right\},
$$

$$
\phi(A)^{\#} \cap\left\{\left[\begin{array}{ll}
0 & x \\
0 & y
\end{array}\right]: x, y \in \mathbb{C}\right\}=\operatorname{span}\left\{E_{12}\right\}
$$


we have

$$
\phi\left(E_{i j}\right) \in \operatorname{span}\left\{E_{12}\right\}, \quad i, j=1,2 .
$$

The treatment is the same if

$$
\phi\left(M_{2}\right) \subset\left\{\left[\begin{array}{ll}
y & x \\
0 & 0
\end{array}\right]: x, y \in \mathbb{C}\right\} .
$$

We now turn to the second part of the plan. Consider the three linear functionals on $M_{2}$ defined by

$$
f_{1}(X)=\phi(X)_{11}-\phi(X)_{22}, \quad f_{2}(X)=\phi(X)_{12}, \quad f_{3}(X)=\phi(X)_{11}
$$

(where $\phi(X)_{i j}$ denotes the $(i, j)$-entry of $\left.\phi(X)\right)$, and the following two functions from $M_{2}$ to $\mathbb{C}$ : let $g_{1}(X)=\operatorname{det} X$ and let $g_{2}(X)$ be the discriminant of the characteristic polynomial of $X$. Denote by $W$ the kernel of $f_{1}$. Let

$$
\mathcal{F}_{i}=\left\{X \in W: f_{i}(X) \neq 0\right\}
$$

for $i=2$ and $i=3$. Similarly, let

$$
\mathcal{G}_{i}=\left\{X \in W: g_{i}(X) \neq 0\right\}
$$

for $i=1$ and $i=2$. If we can reduce our proof to the case when $\mathcal{F}_{2}, \mathcal{F}_{3}, \mathcal{G}_{1}$, and $\mathcal{G}_{2}$ are all nonempty, then they are all dense and open in $W$ (if the set $\mathcal{G}_{i}$ is nonempty, then it is dense in $W$ as a nonzero polynomial on $W$ cannot vanish on an open subset of $W$ ), and therefore their intersection contains a matrix $B$ which has all the desired properties.

If $\mathcal{F}_{2}$ is empty, there is a scalar $c_{2}$ such that $f_{2}=c_{2} f_{1}$, or equivalently,

$$
\phi\left(M_{2}\right) \subset\left\{\left[\begin{array}{cc}
x & c_{2}(x-y) \\
0 & y
\end{array}\right]: x, y \in \mathbb{C}\right\},
$$

so that every member of $\phi\left(M_{2}\right)$ is diagonalizable, a contradiction.

If $\mathcal{F}_{3}$ is empty, then similarly, we have one of the following three possibilities:

$$
\begin{aligned}
& \phi\left(M_{2}\right) \subset\left\{\left[\begin{array}{ll}
0 & x \\
0 & y
\end{array}\right]: x, y \in \mathbb{C}\right\}, \\
& \phi\left(M_{2}\right) \subset\left\{\left[\begin{array}{ll}
y & x \\
0 & 0
\end{array}\right]: x, y \in \mathbb{C}\right\}, \\
& \phi\left(M_{2}\right) \subset\left\{\left[\begin{array}{cc}
x & y \\
0 & k x
\end{array}\right]: x, y \in \mathbb{C}\right\},
\end{aligned}
$$

where $k$ is a nonzero constant. The first two cases have already been treated. So we assume the third case. If $k=1$, the range of $\phi$ is commutative. So, we will assume that $k \neq 1$. Then because $\phi(I) \in \mathbb{C} I$, we have $\phi(I)=0$. It follows that the diagonal entries of $A$ satisfy $\alpha \neq \beta$. If $\phi(A)$ has nonzero diagonal entries, then $B=A$ has all the desired properties. Hence, we may 
assume that

$$
\phi(A)=\phi\left(\left[\begin{array}{ll}
\alpha & 0 \\
0 & \beta
\end{array}\right]\right)=\left[\begin{array}{ll}
0 & 1 \\
0 & 0
\end{array}\right]
$$

and therefore,

$$
\phi\left(\left[\begin{array}{ll}
\lambda & 0 \\
0 & \mu
\end{array}\right]\right)=\left[\begin{array}{cc}
0 & \tau(\lambda-\mu) \\
0 & 0
\end{array}\right], \quad \lambda, \mu \in \mathbb{C},
$$

for some nonzero $\tau \in \mathbb{C}$. If $\phi\left(E_{12}\right)$ and $\phi\left(E_{21}\right)$ both belong to the linear span of $E_{12}$, the range of $\phi$ is commutative. So, assume that one of them, say $\phi\left(E_{12}\right)$, has nonzero diagonal entries:

$$
\phi\left(E_{12}\right)=\left[\begin{array}{cc}
p & q \\
0 & k p
\end{array}\right]
$$

for some $p, q \in \mathbb{C}$ with $p \neq 0$. Because the matrices

$$
\left[\begin{array}{ll}
0 & 1 \\
0 & 0
\end{array}\right] \text { and }\left[\begin{array}{cc}
z & 1 \\
0 & -z
\end{array}\right]
$$

anti-commute for every $z \in \mathbb{C}$, their images

$$
\left[\begin{array}{cc}
p & q \\
0 & k p
\end{array}\right] \text { and }\left[\begin{array}{cc}
p & q+2 \tau z \\
0 & k p
\end{array}\right]
$$

quasi-commute for every $z \in \mathbb{C}$. The diagonal entries of these two matrices are all nonzero, and therefore they actually commute, a contradiction.

Since $W$ is at least three-dimensional, $\mathcal{G}_{1}$ cannot be empty; otherwise $M_{2}$ would contain a three-dimensional subspace of singular matrices, which is impossible. (See [4].)

Similarly, $M_{2}$ does not contain a three-dimensional subspace of matrices of the form $c I+N$, where $c \in \mathbb{C}$ and $N^{2}=0$. (See, e.g., [5] and [9].) Therefore $\mathcal{G}_{2}$ is nonempty.

We have reduced the whole problem to the case where $\phi$ maps every diagonalizable matrix to a diagonalizable matrix. We can now apply [8] to conclude that we have one of the three possibilities:

(1) The range of $\phi$ is simultaneously diagonalizable.

(2) There exist an invertible $T \in M_{2}$, a nonzero scalar $c$ and a functional $f$ on $M_{2}$ such that

$$
\phi(A)=c T A T^{-1}+f(A) I, \quad A \in M_{2} .
$$

(3) There exist an invertible $T \in M_{2}$, a nonzero scalar $c$ and a functional $f$ on $M_{2}$ such that

$$
\phi(A)=c T A^{t} T^{-1}+f(A) I, \quad A \in M_{2} .
$$

In the first case, $\phi\left(M_{2}\right)$ is commutative, and we are done. The last two cases are reduced, after a similarity, multiplying by a constant, and a transposi- 
tion, if necessary, to just one case:

$$
\phi(A)=A+f(A) I
$$

As in the proof of Theorem 1.4, we see that $f(A)=\alpha \operatorname{tr} A$ for some scalar $\alpha$. We will complete the proof by showing that $\alpha$ is zero, -1 or $-1 / 2$. Indeed, if $\alpha \notin\{-1,-1 / 2,0\}$, then $E_{12}$ and $\alpha E_{11}-(1+\alpha) E_{22}$ quasi-commute, but $\phi\left(E_{12}\right)=E_{12}$ and $\phi\left(\alpha E_{11}-(1+\alpha) E_{22}\right)=\alpha E_{11}-(1+\alpha) E_{22}-\alpha I=$ $-(1+2 \alpha) E_{22}$ do not.

\section{References}

[1] M. Brešar, Commuting traces of biadditive mappings, commutativity-preserving mappings and Lie mappings, Trans. Amer. Math. Soc. 335 (1993), 525-546.

[2] M. Brešar and P. Šemrl, On bilinear maps on matrices with applications to commutativity preservers, J. Algebra 301 (2006), 803-837.

[3] J. A. Brooke, P. Busch and D. B. Pearson, Commutativity up to a factor of bounded operators in complex Hilbert space, Proc. R. Soc. London Ser. A Math. Phys. Eng. Sci. 458 (2002), 109-118.

[4] P. Fillmore, C. Laurie and H. Radjavi, On matrix spaces with zero determinant, Linear Multilinear Algebra 18 (1985), 255-266.

[5] M. Gerstenhaber, On nilalgebras and linear varieties of nilpotent matrices. I, Amer. J. Math. 80 (1958), 614-622.

[6] O. Holtz, V. Mehrmann and H. Schneider, Potter, Wielandt, and Drazin on the matrix equation $A B=\omega B A$ : New answers to old questions, Amer. Math. Monthly 111 (2004), 655-667.

[7] L. Molnár, Linear maps on matrices preserving commutativity up to a factor, Linear Multilinear Algebra, to appear.

[8] M. Omladič and P. Šemrl, Preserving diagonalisability, Linear Algebra Appl. 285 (1998), 165-179.

[9] —, - Matrix spaces with bounded number of eigenvalues, ibid. 249 (1996), 29-46.

[10] P. Šemrl, Commutativity preserving maps, ibid., to appear.

[11] J. Yang and H. K. Du, A note on commutativity up to a factor of bounded operators, Proc. Amer. Math. Soc. 132 (2004), 1713-1720.

Department of Pure Mathematics

University of Waterloo

200 University Avenue West

Waterloo, ON, Canada N2L 3G1

E-mail: hradjavi@math.uwaterloo.ca
Department of Mathematics University of Ljubljana Jadranska 19

SI-1000 Ljubljana, Slovenia E-mail: peter.semrl@fmf.uni-lj.si

Received May 16, 2007 